\title{
OUR MILKY WAY AS A PURE-DISK GALAXY-A CHALLENGE FOR GALAXY FORMATION
}

\author{
Juntai Shen ${ }^{1,2}$, R. Michael Rich ${ }^{3}$, John Kormendy ${ }^{2}$, Christian D. Howard ${ }^{3,4}$, Roberto De Propris $^{5}$, \\ AND ANDREA KUNDER ${ }^{5}$ \\ ${ }^{1}$ Key Laboratory for Research in Galaxies and Cosmology, Shanghai Astronomical Observatory, Chinese Academy of Sciences, 80 Nandan Road, \\ Shanghai 200030, China \\ 2 Department of Astronomy, The University of Texas at Austin, 1 University Station, C1400, Austin, TX 78712, USA \\ ${ }^{3}$ Department of Physics and Astronomy, University of California at Los Angeles, Los Angeles, CA, USA \\ ${ }^{4}$ NASA Ames Research Center, MS 211-3 Moffett Field, CA 94035, USA \\ ${ }^{5}$ Cerro Tololo Inter-American Observatory, Casilla 603, La Serena, Chile \\ Received 2010 April 22; accepted 2010 July 19; published 2010 August 12
}

\begin{abstract}
Bulges are commonly believed to form in the dynamical violence of galaxy collisions and mergers. Here, we model the stellar kinematics of the Bulge Radial Velocity Assay (BRAVA) and find no sign that the Milky Way contains a classical bulge formed by scrambling pre-existing disks of stars in major mergers. Rather, the bulge appears to be a bar seen somewhat end-on, as hinted from its asymmetric boxy shape. We construct a simple but realistic $N$-body model of the Galaxy that self-consistently develops a bar. The bar immediately buckles and thickens in the vertical direction. As seen from the Sun, the result resembles the boxy bulge of our Galaxy. The model fits the BRAVA stellar kinematic data covering the whole bulge strikingly well with no need for a merger-made classical bulge. The bar in our best-fit model has a half-length of $\sim 4 \mathrm{kpc}$ and extends $20^{\circ}$ from the Sun-Galactic center line. We use the new kinematic constraints to show that any classical bulge contribution cannot be larger than $\sim 8 \%$ of the disk mass. Thus, the Galactic bulge is a part of the disk and not a separate component made in a prior merger. Giant, pure-disk galaxies like our own present a major challenge to the standard picture in which galaxy formation is dominated by hierarchical clustering and galaxy mergers.
\end{abstract}

Key words: Galaxy: bulge - Galaxy: kinematics and dynamics - galaxies: kinematics and dynamics

\section{INTRODUCTION}

Astronomers commonly make the Copernican assumption that our Milky Way is in no way unusual; then they can exploit the fact that we live in it to study galaxy formation in special detail. Past assumptions about our Galactic bulge grew out of our developing understanding of galaxy formation. It is well known that spiral galaxies consist of three main components, an invisible dark matter halo, an embedded flat disk, and a central bulge. The bulge of our Galaxy is $>99 \%$ made of stars that are at least 5 Gyr old (Clarkson et al. 2008) with a wide range of metal abundances (McWilliam \& Rich 1994; Fulbright et al. 2006; Zoccali et al. 2008). In this respect and many others, big bulges are similar to (diskless) elliptical galaxies. The formation of ellipticals is well understood. Hierarchical gravitational clustering of initial fluctuations in the cosmological density results in galaxy collisions and mergers that scramble flat disks into rounder ellipticals (Toomre 1977; White \& Rees 1978; Steinmetz \& Navarro 2002; Nakasato \& Nomoto 2003). Significant energy has been invested in developing this very successful theory of galaxy formation, and it was natural to think that our Galactic bulge is a product of it. There is little danger that the picture is fundamentally wrong (Binney 2004).

But it is incomplete. The theme of this Letter is that our Galactic bulge is indeed normal but that it is prototypical of different formation processes than are usually assumed. A complementary suite of evolution processes shapes isolated galaxies. They evolve by rearranging energy and angular momentum; this grows central components that masquerade as classical bulges but that formed directly out of disks without any collisions (Kormendy 1993; Kormendy \& Kennicutt 2004). To distinguish them from merger remnants, we call them "pseudobulges." They come in two varieties. Some are flattened; they are grown out of disk gas transported inward by nonaxisymmetries such as bars. Another variety is recognized only in edge-on galaxies. When bars form out of disks, they buckle vertically and heat themselves into thickened structures that look box-shaped when seen edge-on (Combes \& Sanders 1981; Combes et al. 1990; Raha et al. 1991). Our Galaxy contains such a box-shaped (Maihara et al. 1978; Weiland et al. 1994; Dwek et al. 1995) pseudobulge (Kormendy \& Kennicutt 2004).

The identification of this boxy structure as an edge-on bar is particularly compelling because infrared imagery shows a parallelogram-shaped distortion (Maihara et al. 1978; Weiland et al. 1994; Dwek et al. 1995) that is naturally explained as a perspective effect: the near end of the bar is closer to us than the far end. Consequently its vertical extent is greater on the near side than on the far side (Blitz \& Spergel 1991). Zhao (1996) developed the first rapidly rotating bar model that fitted this distortion. Zhao's model was based on the Schwarzschild (1979) orbit superposition technique, so it was self-consistent and in steady state, but it did not evolve into that state from plausible initial conditions. Also, little stellar kinematic data were available to constrain Zhao's steady-state model and early $\mathrm{N}$-body models (Fux 1997, 1999; Sevenster et al. 1999), and subsequent radial velocity data from a survey of planetary nebulae, although compared with a range of dynamical models (Beaulieu et al. 2000), led to only limited conclusions because of the small numbers and uncertain population membership of the planetary nebulae.

In this Letter, we numerically simulate the self-consistent formation of a bar that buckles naturally into a thickened state, and we scale that model to fit new kinematic data on bulge rotation and random velocities. The radial velocity observations 

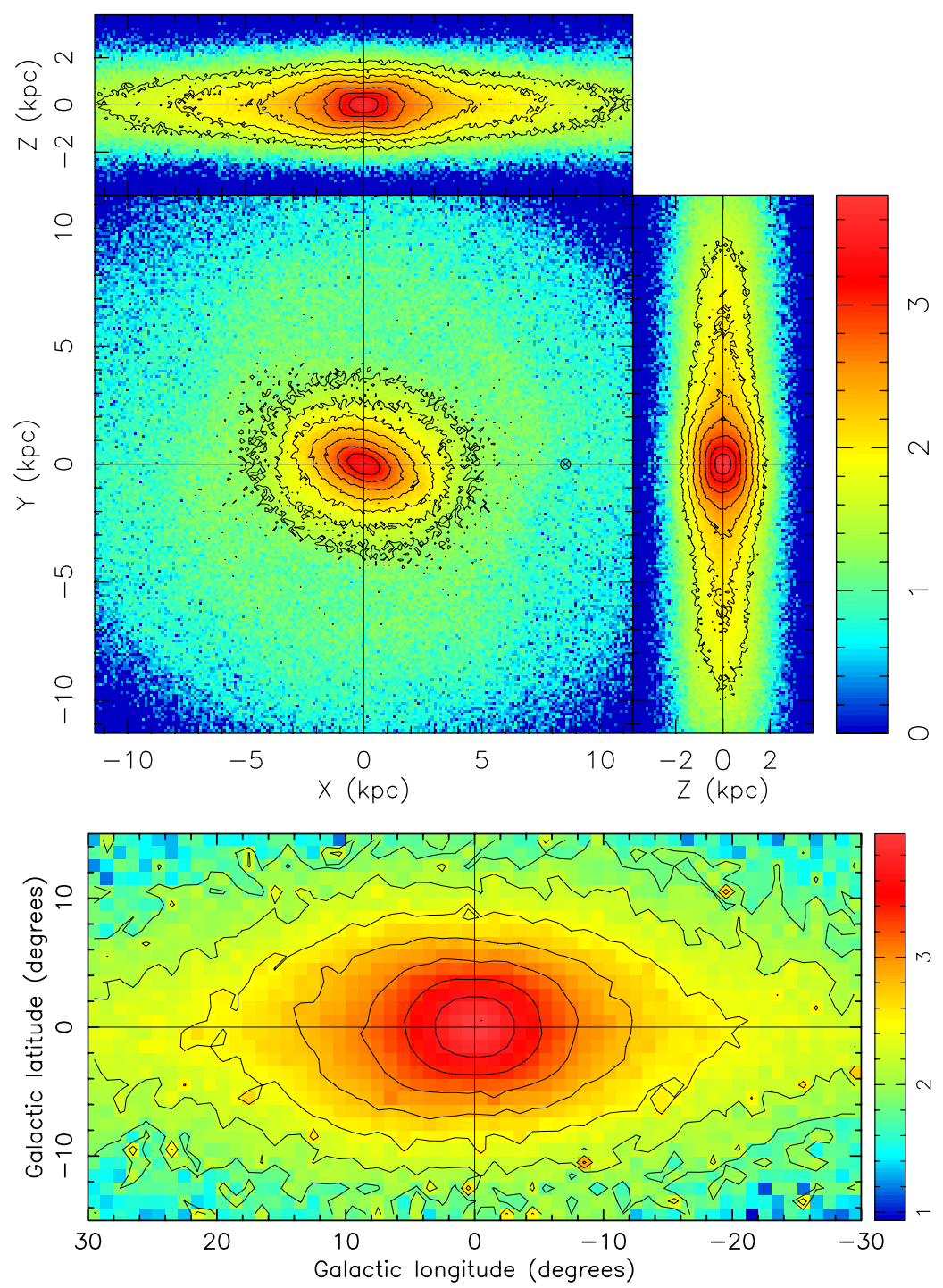

Figure 1. Upper three panels: face-on and side-on views of the surface density of our best-fitting model as seen from far away. The Sun's position $8.5 \mathrm{kpc}$ from the Galactic center is marked along the $+x$ axis. The Galaxy rotates clockwise as seen in the face-on projection. Bottom panel: model surface brightness map in Galactic coordinates as seen from the Sun's location. Our perspective makes the box-shaped, edge-on bar look taller on its nearer side. The Galactic boxy bulge is observed to be similarly distorted.

are provided by the Bulge Radial Velocity Assay (BRAVA; Rich et al. 2007; Howard et al. 2008). This is a spectroscopic survey of the stellar radial velocities of M-type giant stars whose population membership in the bulge is well established. These giants provide most of the $2 \mu \mathrm{m}$ radiation whose box-shaped light distribution motivates bar models. BRAVA emphasizes measurements in two strips at latitude $b=-4^{\circ}$ and $b=-8^{\circ}$ and at longitude $-10^{\circ}<l<+10^{\circ}$. A strip along the minor axis $\left(l \equiv 0^{\circ}\right)$ has also been observed. We use nearly 5000 stellar radial velocities in this Letter. A preliminary analysis of data found strong cylindrical rotation (Howard et al. 2009) consistent with an edge-on, bar-like pseudobulge although a precise fit of a bar model to the data was not available. This success leads us here to construct a fully evolutionary $N$-body model that we can fit to the radial velocity data.

\section{MODEL SETUP}

We use a cylindrical particle-mesh code (Sellwood \& Valluri 1997; Shen \& Sellwood 2004) to build fully self-consistent
$\mathrm{N}$-body galaxies. It is well suited to study the evolution of disk galaxies: we model the disk with at least one million particles to provide high particle resolution near the center where the density is high. We try to construct the simplest self-consistent $N$-body models that fit the BRAVA data, avoiding contrived models with too many free parameters. Initially, they contained only an unbarred disk and a dark halo. The profile of the Galactic halo is poorly constrained observationally; we adopt a rigid pseudoisothermal halo potential $\Phi=\frac{1}{2} V_{\mathrm{c}}^{2} \ln \left(1+\frac{r^{2}}{R_{\mathrm{c}}^{2}}\right)$. Here $V_{\mathrm{c}} \sim$ $250 \mathrm{~km} \mathrm{~s}^{-1}$ is the asymptotic circular-orbit rotation velocity at infinity, and $R_{\mathrm{c}}=15 \mathrm{kpc}$ is the core radius inside which the potential is effectively constant. This halo gives a nearly flat rotation curve of $\sim 220 \mathrm{~km} \mathrm{~s}^{-1}$ between 5 and $20 \mathrm{kpc}$. A simple halo form allows us to run many simulations quickly; this is important for a parameter search such as the present one. A rigid halo also omits dynamical friction on the bar, but the central density of the cored halo we adopt is low enough so that friction will be very mild (e.g., Debattista \& Sellwood 2000; Athanassoula \& Misiriotis 2002). More importantly, we 




Figure 2. Top: mean velocity and velocity dispersion profiles of the best-fitting model (black lines) compared to all available kinematic observations. The left two panels are for the Galactic latitude $b=-4^{\circ}$ strip; the middle two panels are for the $b=-8^{\circ}$ strip; and the right two panels are for the $l=0^{\circ}$ minor axis. The black diamonds and their error bars are the BRAVA data; the green diamonds are for M-type giant stars (Rangwala et al. 2009), and the red triangles are the data on red clump giant stars (Rangwala et al. 2009). This is the first time that a single dynamical model has been compared with data of such quality. The agreement is striking.

are mainly interested in the bulge, which is embedded well interior to $R_{\mathrm{c}}$. So, the exact profile of the dark halo at large radii is not critical. We will explore more sophisticated halos in a future study.

\section{RESULTS AND DISCUSSIONS}

In our models, a bar develops self-consistently from the initially unbarred, thin disk. Bar formation enhances the radial streaming motions of disk particles, so the radial velocity dispersion quickly grows much bigger than the vertical one. Consequently, the disk buckles vertically out of the plane like a fire hose; this is the well-known buckling or corrugation instability (Toomre 1966; Combes et al. 1990; Raha et al. 1991). It raises the vertical velocity dispersion and increases the bar's thickness. This happens on a short dynamical timescale and saturates in a few hundred million years. The central part of the buckled bar is elevated well above the disk mid-plane and resembles the peanut morphology of many bulges including the one in our Galaxy (Combes et al. 1990; Raha et al. 1991).

Out of a large set of $\mathrm{N}$-body models, we find the one that best matches our BRAVA kinematic data after suitable mass scaling. The barred disk evolved from a thin exponential disk that contains $M_{\mathrm{d}}=4.25 \times 10^{10} M_{\odot}$, about $55 \%$ of the total mass at the truncation radius (5 scale lengths). The scale length and scale height of the initial disk are $\sim 1.9 \mathrm{kpc}$ and $0.2 \mathrm{kpc}$, respectively. The disk is rotationally supported and has a Toomre- $Q$ of 1.2. The amplitude of the final bar is intermediate between the weakest and strongest bars observed in galaxies. The bar's minor-to-major axial ratio is about 0.5-0.6, and its half-length is $\sim 4 \mathrm{kpc}$. Figure 1 (top three panels) shows face-on and side-on views of the projected density of the best-fitting model. A distinctly peanut-shaped bulge is apparent in the edge-on projection. Figure 1 (bottom panel) shows the surface brightness distribution in Galactic coordinates as seen from the Sun's vantage point. Nearby disk stars dilute the peanut shape, but the bar still looks boxy. Moreover, from close-up,

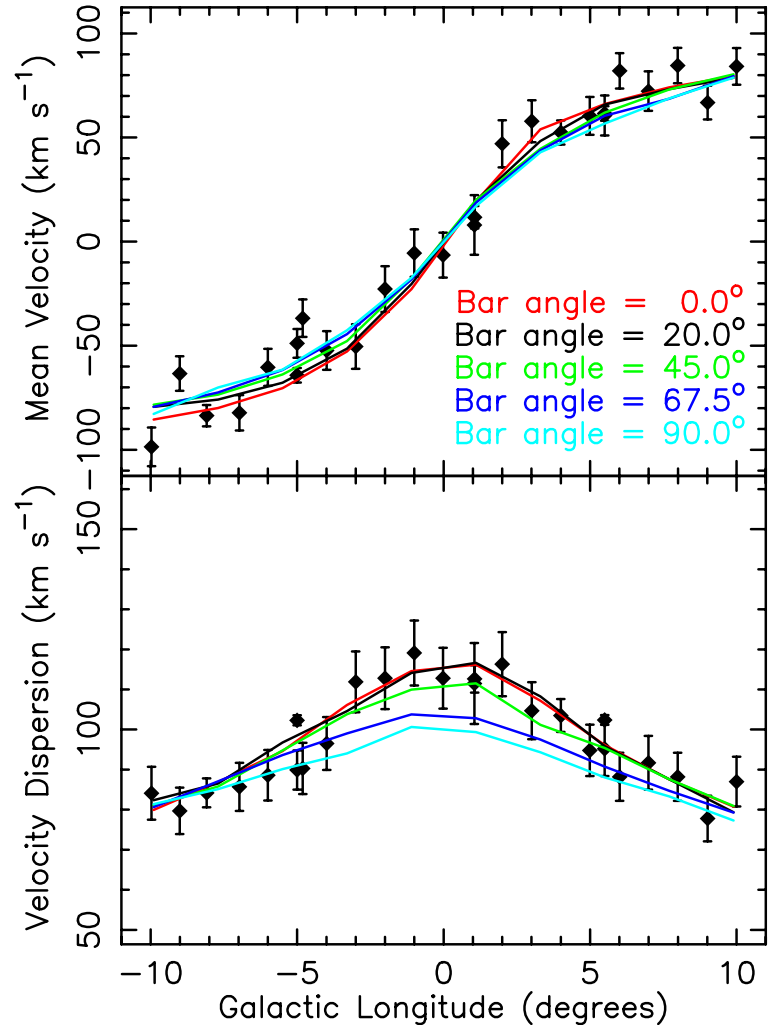

Figure 3. Model results compared to the data from the $b=-4^{\circ}$ major-axis strip as we vary the bar angle relative to the line that connects the Sun to the Galactic center. The red, black, green, blue, and cyan curves are the model results for bar angles of $0^{\circ}, 20^{\circ}$ (the adopted best-fit value), $45^{\circ}, 67^{\circ} .5$, and $90^{\circ}$, respectively. All data points are plotted as black diamonds regardless of source. Clearly bar angles of $0^{\circ}$ and $20^{\circ}$ are favored over larger values.

an asymmetry in the longitudinal direction is apparent; this means that the bar cannot be aligned with the direction from the Sun to the Galactic center. Rather, its near end is at positive 


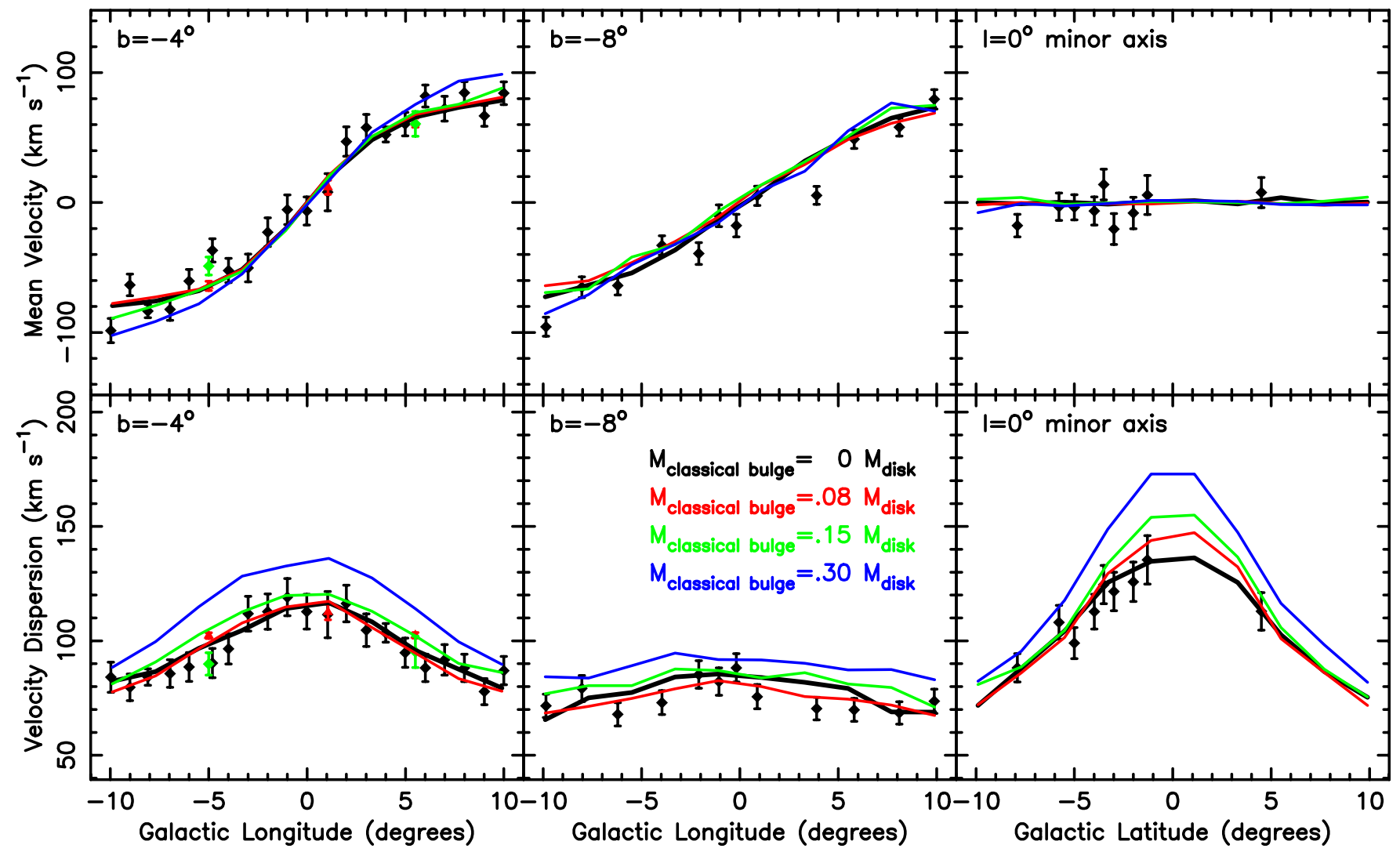

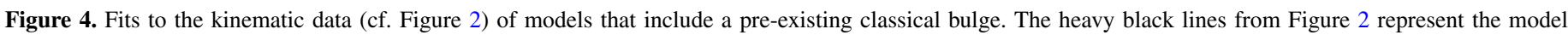


Including a classical bulge significantly worsens the model fits to the data, especially along the minor axis.

Galactic longitude, so it looks taller in that quadrant, and it extends farther from the Galactic center on the near side than on the far side. Both the boxy shape and the asymmetry are in good agreement with the morphology revealed by the COBE satellite near-infrared images (Weiland et al. 1994; Dwek et al. 1995).

Figure 2 compares the best-fitting model kinematics (solid lines) with the mean velocity and velocity dispersion data from the BRAVA and other surveys (Rangwala et al. 2009). All velocities presented here have been converted to Galactocentric values (the line-of-sight velocity that would be observed by a stationary observer at the Sun's position). For the first time, our model is able to simultaneously match the mean velocities and velocity dispersions along two Galactic latitudes $\left(-4^{\circ}\right.$ and $\left.-8^{\circ}\right)$ and along the minor axis.

Figure 3 constrains the angle between the bar and the line that connects the Sun to the Galactic center. It compares the model results with the data in the $b=-4^{\circ}$ major-axis strip as we vary the above angle. Clearly, the smallest bar angles give the best match to the velocity dispersions. Intriguingly, we find that the velocity dispersions provide much stronger constraints than the mean velocity profile. A bar angle of $0^{\circ}$ also matches the kinematics well. However, the photometric asymmetry excludes a bar that is pointed at the Sun. We therefore conclude that the overall best-fitting model has a bar angle of $\sim 20^{\circ}$. Other studies converged on a similar bar angle (Stanek et al. 1997; Freudenreich 1998; Fux 1997, 1999; Bissantz \& Gerhard 2002). The excellent match to the data in Figures 1-3 strongly supports the suggestion that the boxy pseudobulge of the Milky Way is an edge-on, buckled bar that evolved from a cold, massive disk.
The thickened disk in the pseudobulge-forming process may have contributed to the thick disk of the Milky Way, as hinted from chemical similarities of Galactic bulge and local thick disk stars (Alves-Brito et al. 2010; Bensby et al. 2010).

The model in Figures 1-3 contains no classical bulge component. Could a small classical bulge also be present? Could it have been spun up by the formation of a bar, flattened thereby and made hard to detect? To constrain such multi-component models with BRAVA kinematics, we also constructed models with a pre-existing classical bulge. The distribution function for the live classical bulge component was generated iteratively (Debattista \& Sellwood 2000) to ensure that both the disk and the classical bulge were initially in equilibrium, and we required that the bulge parameters are close to the fundamental plane for classical bulges and ellipticals (Kormendy et al. 2009). The setup of the disk is the same as in the disk-only model. We show in Figure 4 that the inclusion of a classical bulge-one widely thought to be typical of Sbc spiral galaxies like our own-greatly worsens the model fit to the data. The degradation is especially obvious along the Galaxy's minor axis. Including a classical bulge with just $8 \%$ of the disk mass considerably worsens the fit of the model to the data. Our models rule out that the Milky Way has a significant classical bulge whose mass is $>\sim 15 \%$ of the disk mass.

Could a smaller, merger-built bulge hide inside the boxy bar? The only result that we are aware of that might point to such a conclusion is the observed drop in stellar metal abundances with increasing height above the Galactic plane (Zoccali et al. 2008; Zoccali 2010). Zoccali et al. (2008) argue that this means that the bulge must consist of both a classical and an edge-on 
bar component. However, no kinematic gradient or transition corresponding to the abundance gradient is observed. Moreover, an abundance gradient can be produced within the context of secular pseudobulge formation if some of the vertical thickening is produced by resonant heating of stars that scatter off the bar (Pfenniger \& Norman 1990). If the most metal-poor stars are also the oldest stars, then they have been scattered for the longest time and now reach the greatest heights.

Our results have important implications for galaxy formation. We demonstrate that the boxy pseudobulge is not a separate component of the Galaxy but rather is an edge-on bar. Bars are parts of disks. To be sure, the stars in our Galactic bar are older than most disk stars. But those stars could have formed over a short period of time long before the bar structure formed (Wyse 1999; Freeman 2008). Their old age (Zoccali et al. 2003; Fulbright et al. 2007) is therefore not an argument against the internal secular evolution model. Our kinematic observations show no sign that the Galaxy contains a significant mergermade, "classical" bulge. So, from a galaxy formation point of view, we live in a pure-disk galaxy. Our Galaxy is not unusual: it is very similar to another giant edge-on galaxy with a boxy bulge, NGC 4565. Kormendy \& Barentine (2010) recently showed that NGC 4565 does not contain even a small classical bulge component and that it is therefore another giant, puredisk galaxy that contains no sign of a merger remnant. In fact, giant, pure-disk galaxies are common in environments like our own that are far from rich clusters of galaxies (Kormendy et al. 2010). Classical bulgeless, pure-disk galaxies present an acute challenge to the current picture of galaxy formation in a universe dominated by cold dark matter-growing a giant galaxy via hierarchical clustering ( $V_{\mathrm{c}} \simeq 220 \mathrm{~km} \mathrm{~s}^{-1}$ in the Milky Way) involves so many mergers that it seems almost impossible to avoid forming a substantial classical bulge (Peebles \& Nusser 2010; Agertz et al. 2010). How did our Galaxy grow so large with no observational sign that it suffered a major merger after the time 9-10 Gyr ago (e.g., Winget \& Kepler 2008) when the first disk stars formed?

We thank Karl Gebhardt for helpful discussions and warm support on this project. We also acknowledge support from the US National Science Foundation under grants AST-0607490 (J.K.) and AST-0709479 (R.M.R.).

\section{REFERENCES}

Agertz, O., Teyssier, R., \& Moore, B. 2010, arXiv:1004.0005

Alves-Brito, A., Meléndez, J., Asplund, M., Ramírez, I., \& Yong, D. 2010, A\&A, 513, A35

Athanassoula, E., \& Misiriotis, A. 2002, MNRAS, 330, 35

Beaulieu, S. F., Freeman, K. C., Kalnajs, A. J., Saha, P., \& Zhao, H. 2000, AJ, 120,855
Bensby, T., Alves-Brito, A., Oey, M. S., Yong, D., \& Meléndez, J. 2010, A\&A, 516, L13

Binney, J. 2004, in IAU Symp. 220, Dark Matter in Galaxies, ed. S. D. Ryder,

D. J. Pisano, M. A. Walker, \& K. C. Freeman (San Francisco, CA: ASP), 3

Bissantz, N., \& Gerhard, O. 2002, MNRAS, 330, 591

Blitz, L., \& Spergel, D. N. 1991, ApJ, 379, 631

Clarkson, W., et al. 2008, ApJ, 684, 1110

Combes, F., Debbasch, F., Friedli, D., \& Pfenniger, D. 1990, A\&A, 233, 82

Combes, F., \& Sanders, R. H. 1981, A\&A, 96, 164

Debattista, V. P., \& Sellwood, J. A. 2000, ApJ, 543, 704

Dwek, E., et al. 1995, ApJ, 445, 716

Freeman, K. C. 2008, in IAU Symp. 245, Formation and Evolution of Galaxy Bulges, ed. M. Bureau, E. Athanassoula, \& B. Barbuy (Cambridge: Cambridge Univ. Press), 3

Freudenreich, H. T. 1998, ApJ, 492, 495

Fulbright, J. P., McWilliam, A., \& Rich, R. M. 2006, ApJ, 636, 821

Fulbright, J. P., McWilliam, A., \& Rich, R. M. 2007, ApJ, 661, 1152

Fux, R. 1997, A\&A, 327, 983

Fux, R. 1999, A\&A, 345, 787

Howard, C. D., Rich, R. M., Reitzel, D. B., Koch, A., De Propris, R., \& Zhao, H. 2008, ApJ, 688, 1060

Howard, C. D., et al. 2009, ApJ, 702, L153

Kormendy, J. 1993, in IAU Symp. 153, Galactic Bulges, ed. H. Dejonghe \& H. J. Habing (Dordrecht: Kluwer), 209

Kormendy, J., \& Barentine, J. C. 2010, ApJ, 715, L176

Kormendy, J., Drory, N., Bender, R., \& Cornell, M. E. 2010, ApJ, submitted

Kormendy, J., Fisher, D. B., Cornell, M. E., \& Bender, R. 2009, ApJS, 182, 216

Kormendy, J., \& Kennicutt, R. C. 2004, ARA\&A, 42, 603

Maihara, T., Oda, N., Sugiyama, T., \& Okuda, H. 1978, PASJ, 30, 1

McWilliam, A., \& Rich, R. M. 1994, ApJS, 91, 749

Nakasato, N., \& Nomoto, K. 2003, ApJ, 588, 842

Peebles, P. J. E., \& Nusser, A. 2010, Nature, 465, 565

Pfenniger, D., \& Norman, C. 1990, ApJ, 363, 391

Raha, N., Sellwood, J. A., James, R. A., \& Kahn, F. D. 1991, Nature, 352, 411

Rangwala, N., Williams, T. B., \& Stanek, K. Z. 2009, ApJ, 691, 1387

Rich, R. M., Reitzel, D. B., Howard, C. D., \& Zhao, H. 2007, ApJ, 658, L29

Schwarzschild, M. 1979, ApJ, 232, 236

Sellwood, J. A., \& Valluri, M. 1997, MNRAS, 287, 124

Sevenster, M., Saha, P., Valls-Gabaud, D., \& Fux, R. 1999, MNRAS, 307, 584

Shen, J., \& Sellwood, J. A. 2004, ApJ, 604, 614

Stanek, K. Z., Udalski, A., Szymanski, M., Kaluzny, J., Kubiak, M., Mateo, M., \& Krzeminski, W. 1997, ApJ, 477, 163

Steinmetz, M., \& Navarro, J. F. 2002, New Astron., 7, 155

Toomre, A. 1966, in Geophysical Fluid Dynamics, Notes on the 1966 Summer Study Program at the Woods Hole Oceanographic Institution, Ref. No. 66-46 (Woods Hole, MA: Woods Hole Oceanographic Inst.), 111

Toomre, A. 1977, in Evolution of Galaxies and Stellar Populations, ed. B. M. Tinsley \& R. B. Larson (New Haven, CT: Yale Univ. Obs.), 401

Weiland, J. L., et al. 1994, ApJ, 425, L81

White, S. D. M., \& Rees, M. J. 1978, MNRAS, 183, 341

Winget, D. E., \& Kepler, S. O. 2008, ARA\&A, 46, 157

Wyse, R. F. G. 1999, in The Formation of Galactic Bulges, ed. C. M. Carollo, H. C. Ferguson, \& R. F. G. Wyse (Cambridge: Cambridge Univ. Press), 195 Zhao, H. S. 1996, MNRAS, 283, 149

Zoccali, M. 2010, in IAU Symp. 265, Chemical Abundances in the Universe: Connecting First Stars to Planets, ed. K. Cunha, M. Spite, \& B. Barbuy (Cambridge: Cambridge Univ. Press), 271

Zoccali, M., Hill, V., Lecureur, A., Barbuy, B., Renzini, A., Minniti, D., Gómez, A., \& Ortolani, S. 2008, A\&A, 486, 177

Zoccali, M., et al. 2003, A\&A, 399, 931 